\title{
Synthesis, properties, and optical applications of noble metal nanoparticle-biomolecule conjugates
}

\author{
ZHANG YuJuan ${ }^{1}$, HUANG Rao $^{1}$, ZHU XianFang $^{1,2^{*}}$, WANG LianZhou ${ }^{1,2} \&$ WU ChenXu ${ }^{1}$ \\ ${ }^{1}$ China-Australia Joint Laboratory for Functional Nanomaterials and Physics Department, Xiamen University, Xiamen 361005, China; \\ ${ }^{2}$ ARC Centre of Excellence for Functional Nanomaterials, University of Queensland, St Lucia, Brisbane, Qld 4072, Australia
}

Received November 19, 2010; accepted August 11, 2011

\begin{abstract}
Noble metal nanoparticles, such as gold or silver nanoparticles and nanorods, exhibit unique photonic, electronic and catalytic properties. Functionalization of noble metal nanoparticles with biomolecules (e.g., protein and DNA) produces systems that possess numerous applications in catalysis, delivery, therapy, imaging, sensing, constructing nanostructures and controlling the structure of biomolecules. In this paper, the recent development of noble metal nanoparticle-biomolecule conjugates is reviewed from the following three aspects: (1) synthesis of noble metal nanoparticle-biomolecule systems by electrostatic adsorption, direct chemisorption of thiol derivatives, covalent binding through bifunctional linkers and specific affinity interactions; (2) the photonic properties and bioactivation of noble metal nanoparticle-biomolecule conjugates; and (3) the optical applications of such systems in biosensors, and medical imaging, diagnosis, and therapy. The conjugation of $\mathrm{Au}$ and $\mathrm{Ag}$ nanoparticles with biomolecules and the most recent optical applications of the resulting systems have been focused on.
\end{abstract}

noble metal nanoparticle, biomolecule, integration

Citation: Zhang Y J, Huang R, Zhu X F, et al. Synthesis, properties, and optical applications of noble metal nanoparticle-biomolecule conjugates. Chin Sci Bull, 2012, 57: 238-246, doi: 10.1007/s11434-011-4747-x

With the development of nanotechnology in biology and medicine, the demand to integrate nanoparticles with biomolecules to form conjugated or hybrid systems has increased in recent years. This is because such structures are versatile in applications including catalysis [1], delivery [2], therapy [3], imaging [4], sensing [5], constructing nanostructures [6] and controlling the structure of biomolecules [7].

Metal nanoparticles [4,5,8] are widely used to construct structures that possess unique electric, photonic and catalytic properties such as local surface plasmon resonance (LSPR) [7,9-11], surface-enhanced Raman scattering (SERS) [12], and surface-enhanced fluorescence (SEF) $[13,14]$. There are two fundamental strategies used to prepare nanoparticles: bottom-up and top-down. The bottomup approach is a basic technique to prepare the metal nanoparticles by reducing their ions and the growth of the nano-

*Corresponding author (email: zhux @xmu.edu.cn) particles is usually stopped by an agent such as a surfactant or stabilizer. Bottom-up techniques include chemical reduction [15-17], photochemical reduction [18], electrochemical reduction [19], and templating [20] and thermal methods [21,22]. Bottom-up methods allow large-scale synthesis of the nanoparticles, but an obvious disadvantage is that the nanoparticles are usually of non-uniform size and shape compared with those produced by a top-down method. The top-down approach involves removing material from the bulk substrate to leave behind the desired nanostructures. Common top-down methods include photolithography, electron beam lithography, and nanosphere lithography (NSL) [23,24].

Many biomolecules including proteins/enzymes/oligopeptides [7,25], antibody/antigens [1,26], biotin/streptavidin [23,24], and DNA/oligonucleotides/aptamers [6] have been immobilized on the surface of nanoparticles to form noble metal nanoparticle-biomolecule conjugates. The similar size of nanoparticles and biomolecules makes them rela- 
tively easy to integrate.

The synthesis and applications of biomolecule-nanoparticle hybrid systems have been reviewed [27,28]. There have also been a number of articles that review different specific applications of biomolecule-nanoparticle hybrids, such as biosensing [29-34], probing cells [35], delivery [36] and diagnosis [37,38]. For example, Katz et al. [27] reviewed advances in the synthesis of biomolecule (proteins or DNA)-nanoparticle (metal or semiconductor nanoparticles) conjugates as well as their application. Aubin-Tam et al. [28] reviewed studies of the conjugation of protein and nanoparticles using a linker species. In addition, many specific applications have also been reviewed: biomoleculenanoparticle conjugates for the assembly of electrochemical biosensors [29-32], noble metal nanoparticle aggregates as tags for probing cells [35], gold nanoparticles as non-toxic carriers for drug and gene delivery [36], and gold nanoparticles for application in clinical diagnosis [37]. The development of biomolecule-nanoparticle systems has been very rapid, and many new materials have been reported. As a result, it is very imperative for a review of recent research results including new synthesis methods, optical properties and applications. In this paper, the latest studies on the synthesis, optical and bioactive properties, and optical applications of nanoparticle-biomolecule systems are reviewed. Noble metal nanoparticle-biomolecule systems such as $\mathrm{Au}$ or Ag-biomolecule conjugates, and their related optical applications in biology and medicine are the focus of this review.

\section{Synthesis of noble metal nanoparticle- biomolecule conjugates}

Conjugation of noble metal nanoparticles with biomolecules has mainly been achieved by one of four major mechanisms: electrostatic adsorption, direct chemisorption of thiol derivatives, covalent binding through bifunctional linkers, and specific affinity interactions. Among these mechanisms, specific affinity interactions can be further classified into sub-categories including biotin-streptavidin binding, antibody-antigen conjugation, and complementary DNA association. These mechanisms are schematically described in Figure 1.

\subsection{Electrostatic adsorption}

Electrostatic adsorption is a physical approach to fix biomolecules on the surface of nanoparticles. The nanoparticles and biomolecules with opposite charges will be to form the nanoparticle-biomolecule system. As shown in Figure 1(a), silver nanoparticles produced by reduction of citrate can be decorated with human serum albumin (HSA) at $\mathrm{pH}$ values higher than the isoelectric point of HSA, which allows more effective binding between the positively charged amino acid side chains of the protein and the negatively charged citrate group of the silver nanoparticles [25].

The electrostatic deposition of biomolecules on nanoparticles such as proteins, enzymes and oligopeptides has recently been extended to multilayer assemblies through the use of layer-by-layer methods [39], which have been widely used to construct multilayer assemblies to enhance the selectivity of multilayer conjugation [40]. In particular, Angelatos et al. [40] used a layer-by-layer coating of colloidal templates with polyelectrolytes to form core-shell particles. They then decomposed the colloidal templates to yield polyelectrolyte multilayer capsules, and loaded macromolecules into these capsules by exploiting the $\mathrm{pH}$-dependence of the permeability of the shell. Finally, they were able to

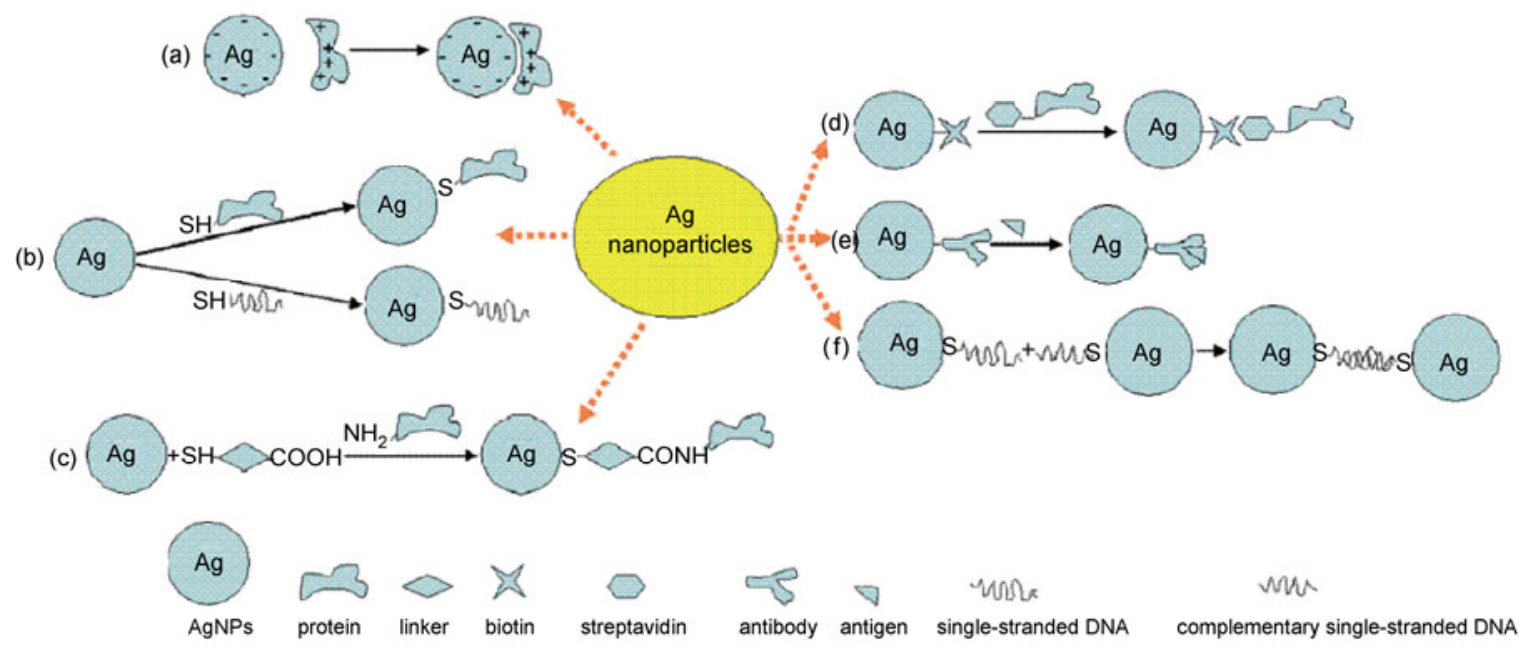

Figure 1 (Color online) Synthesis of biomolecule-nanoparticle systems by different routes: (a) assembly of nanoparticle (NP)-protein conjugates by electrostatic adsorption; (b) conjugation of biomolecules (proteins/DNA) on NPs through direct chemisorption of thiol derivation; (c) covalent binding through bifunctional linkers to form biomolecule-NP hybrids; (d)-(f) specific affinity interactions between biomolecules and NPs including (d) biotin-streptavidin binding, (e) antibody-antigen conjugation, and (f) complementary DNA association. 
render the capsules optically active by infiltrating lightabsorbing gold nanoparticles into the capsule shell through electrostatic adsorption.

Electrostatic adsorption can be used to assemble biomolecules on the surface of nanoparticles. Furthermore, it causes mutual deposition of nanoparticles and biomolecules. For example, 4-(dimethylamino)pyridine-stabilized gold nanoparticles could infiltrate into polyelectrolyte multilayers preassembled on indium tin oxide electrodes, which led to the formation of multilayer films for use as an electrochemical sensor [42].

Electrostatic adsorption is a simple and convenient method to construct nanoparticle-biomolecule systems; however, it possesses some drawbacks. The systems obtained using this method are unstable and will induce a slight change in the conformation of the biomolecules. For example, the integration of $\mathrm{Ag}$ nanoparticles with bovine hemoglobin caused the content of $\alpha$-helices in the protein to decrease from $72.5 \%$ to $66.2 \%$ [43].

\subsection{Direct chemisorption of thiol derivatives}

Direct chemisorption of thiol derivatives on nanoparticles is a simple chemical reaction that occurs between the surface of a nanoparticle and thiol group(s) of a biomolecule (see Figure 1(b)). Thiol groups can be introduced onto the outer part of biomolecules to allow interaction with the surface of nanoparticles. In this situation, the thiol groups in the biomolecules are derived from amino acid residues or are incorporated by chemical methods. Cysteine is usually used to provide thiol groups to bind noble metal nanoparticles with biomolecules. Cysteine residues can assemble on the surface of gold nanoparticles by direct formation of thiol-Au bonds $[44,45]$.

Recently, new applications related to the direct chemisorption of thiol derivatives have appeared. For example, nucleic acids have been attached to nanoparticles through thiol anchor groups [46-49]. Addition of a single-stranded target oligonucleotide ( 30 bases) to a solution containing the appropriate probes caused a polymeric network of $\mathrm{Au}$ nanoparticles to form with a concomitant color change from red to pinkish/purple [49]. Silver nanoparticles functionalized with oligonucleotides and Raman labels coupled with SERS spectroscopy have been used for multiplexed detection of oligonucleotide targets [47]. However, because particle curvature plays an important role in controlling the surface density of DNA on a nanoparticle, and the packing of DNA on nonspherical particles and Au nanorods is inhomogeneous [46].

In contrast to electrostatic adsorption, direct chemisorption of thiol derivatives can produce a more stable noble metal nanoparticle-biomolecule complex because it involves a chemical reaction. However, it still induces a slight change in the conformation of the biomolecule.

\subsection{Covalent binding through bifunctional linkers}

Covalent binding through bifunctional linkers uses bifunctional linkers of low molecular weight to conjugate biomolecules with noble metal nanoparticles (see Figure 1(c)). Such bifunctional linkers contain both anchor groups to attach to the surface of nanoparticles and functional groups that allow covalent binding to the target biomolecule [24,26,50,51].

Recently, a mixed monolayer of 11-mercaptoundecanoic acid (MUA)/6-mercapto-1-hexanol with a molar ratio of 1:3 was used as a bifunctional linker. The monolayer was combined with the surface of silver nanoparticles through its thiol anchor groups and then further conjugated with the protein IgG by the formation of a peptide bond [52]. A monolayer consisting of 1-octanethiol (1-OT)/MUA with a molar ratio of 3:1 has been used to link silver nanoclusters with biotin [23]. Furthermore, it has also been demonstrated that a monolayer of mercaptopropionic acid on gold nanoparticles could provide reactive carboxyl groups that could be modified with biotin [51].

The problems of instability and inactivation caused by nanoparticle/biomolecule integration can be overcome by using bifunctional linkers. An important advantage of this technique it that it retains the stability and activity of biomolecules to allow further conjugation with other biomolecules [5,52]. For example, biotin decorated with silver nanoparticles using a linker have been further linked to streptavidin $[23,24,51]$.

\subsection{Specific affinity interaction}

Nanoparticles decorated with groups that provide sites to bind to biomolecules have been used to produce adducts with specific biomolecules such as streptavidin (see Figure 1(d)), proteins (see Figure 1(e)) and DNA (see Figure 1(f)). Silver nanoparticles functionalized with biotin have been widely used to bind with streptavidin, which can serve as a linker for further conjugation with biomolecules [23,24,51].

In addition, the specific affinity of antibodies for antigens can allow the specific combination of a functionalized nanoparticle with a target biomolecule [5,26,50,52,53]. For example, silver nanoparticles were coated with a monolayer of 1-octanethiol (1-OT)/MUA (molar ratio of 3:1) through the $\mathrm{Ag}-\mathrm{S}$ bond, and then the carboxyl groups of MUA were conjugated to amyloid-derived diffusible ligands (ADDL) [26]. Finally, the anti-ADDL antibody can bind specifically to ADDL that has been decorated on the surface of the silver nanoparticles.

Oligonucleotides have also been used to link nanoparticles with biomolecules. A three-dimensional crystalline assembly of gold nanoparticles can be constructed by exploiting the interaction between single-stranded DNA and its complementary single-stranded DNA that has been linked on the surface of noble metal nanoparticles [6].

Using specific affinity interactions, a specific target can 
be integrated with functionalized noble metal nanoparticles that have been decorated with a specific biomolecule.

\section{Properties of noble metal nanoparticle- biomolecule conjugates}

Compared with the bulk noble metal (such as gold and silver), noble metal nanoparticles show unique photonic, electronic and catalytic properties [54] resulting from their size and shape as well as the dielectric constant of their surrounding environment [55]. Biomolecules decorated on the surface of such nanoparticles can still show activity [26].

\subsection{Photonic properties of noble metal nanoparticle- biomolecule conjugates}

The surface geometry of noble metal nanoparticles and dielectric constants of the surrounding materials both play important roles in controlling the density of the electric field on the surface of nanoparticles. After decoration with biomolecules, the size and shape of a nanoparticle and dielectric constant of the surrounding environment change slightly, which affects the density of the electric field on the surface of the nanoparticle. The corresponding changes in the oscillation frequency of the electrons change optical properties such as absorption and scattering accordingly [54].

(i) Plasmon resonance extinction. A rhombic Ag nanoparticle array prepared by nanosphere lithography as substrate for biosensors has been used to detect the binding signal between biotin and streptavidin [23]. A shift in the plasmon resonance extinction that results from LSPR has been demonstrated in this array. An LSPR biosensor for detecting ADDLs using a sandwich assay composed of an array of triangular silver nanoparticles has been developed to study the oligomerization of amyloid precursors at low concentration [5]. Other examples have also shown that biomolecules can be detected by LSPR because of shifts in the plasmon resonance extinction upon binding to nanoparticles [7,9-11].

(ii) Surface-enhanced Raman scattering. SERS is a technique that allows sensitive and selective detection of analytes at low concentration [56]. There are two effects that have been considered to account for enhancement of the Raman signal at a surface: chemical enhancement [57] and electromagnetic field enhancement [58].

A SERS-based sensor containing a portable spectrometer has been used to detect bacillus spores at the desired limit of detection $\left(\sim 10^{4}\right.$ spores $)$ within $5 \mathrm{~s}$ on a silver substrate [12]. Multiplexed detection of oligonucleotide targets has been performed with gold nanoparticle probes that have been labeled with oligonucleotides and Raman-active dyes. In this system, the gold nanoparticles facilitate the formation of a silver coating that acts as a SERS promoter for dyelabeled particles captured by target molecules and an un- derlying chip in the microarray [47].

(iii) Fluorescence. SEF is caused by the strong electromagnetic field generated on the surface of noble metal nanoparticles [13,14]. Homogeneous silver nanostructures on a glass surface can serve as potential substrates for fluorescence enhancement assays. A monolayer of a fluorescein isothiocyanate (FITC)-HSA conjugate has been formed on glass surfaces covered with silver nanoparticles [59]. In this system, the fluorescence from FITC was enhanced compared with that of the control sample (FITC-HSA monolayer on a bare glass surface).

Furthermore, compared with organic dyes and fluorescent proteins, nanoparticle probes such as fluorescence energy-transfer nanobeads and quantum dots can provide significant advantages in signal brightness, photostability, and multicolor-light emission [14].

\subsection{Bioactivation of noble metal nanoparticle-biomol- ecule conjugates}

Biomolecules decorated on the surface of noble metal nanoparticles are still able to show bioactivity for further reactions through nonspecific adsorption or specific binding. For example, biotin bound to silver nanoparticles can further bind to streptavidin [23,24,51], antibodies decorated on silver nanoparticles can combine with specific antigens $[5,26,50,52,53]$, and single-stranded DNA fixed on gold nanoparticles can still bind to its complementary DNA strand [6].

\section{Optical applications of noble metal nanopar- ticle-biomolecule systems}

Noble metal nanoparticles integrated with biomolecules have numerous applications in catalysis, delivery, therapy, imaging, sensing, constructing nanostructures and controlling the structure of biomolecules. Recent progress of their optical applications in areas such as optical biosensors, imaging, diagnosis and therapy in medicine is focused on in this review.

\subsection{Optical biosensors}

The immobilization of biomolecules on the surface of noble metal nanoparticles provides a route to develop biosensors that operate using properties such as LSPR, SERS, SEF and bioactivation.

Haes et al. [5] developed a nanoscale optical biosensor based on LSPR spectroscopy to monitor the interaction between an antigen (ADDL) and specific anti-ADDL antibodies (Figure 2). Using the sandwich assay format, this biosensor provides quantitative binding information about both the antigen and second antibody detection, allowing the concentration of ADDL to be determined and offering a 
unique analysis of the aggregation mechanisms of this putative Alzheimer's disease pathogen at physiologically relevant monomer concentrations. Monitoring the LSPRinduced shifts from both ADDLs and a second polyclonal anti-ADDL antibody revealed two ADDL epitopes that have binding constants to the specific anti-ADDL antibodies of $7.3 \times 10^{12}$ and $9.5 \times 10^{8} \mathrm{M}^{-1}\left(1 \mathrm{M}=1 \mathrm{~mol} \mathrm{~L}^{-1}\right)$. The analysis of human brain extract and cerebrospinal fluid samples from control and Alzheimer's disease patients showed that the LSPR biosensor can provide new information relevant to the understanding and possible diagnosis of Alzheimer's disease. Zhu and co-workers [23] reported that the LSPR extinction spectra of targets are detected at lower concentration using a nanosensor containing rhombic silver nanoparticles than one containing triangular silver nanoparticles.

Two types transducers to detect the concentration of streptavidin have been compared [60]. These transducers were based on gold nanoparticles and immobilized gold nanoparticles functionalized with polyelectrolyte. It was revealed that the latter can improve the stability of the gold nanoparticles and reduce nonspecific binding with streptavidin.

Furthermore, based on the unique optical properties of gold nanoparticles and the high efficiency of chromatographic separation, a disposable nucleic acid biosensor that could detect nucleic acid samples in 15 min has been developed [61].

Shin et al. have prepared highly uniform noble metal nanoparticle arrays for use as biosensors via block copolymer lithography [62]. Biosensors using the photonic properties of noble metal nanoparticle-biomolecule conjugates have been used to detect targets such as small molecules, biomolecules, cells, and viruses. Using a biosensor to detect targets is a simple and convenient method that has been widely applied in medical and environmental settings.

\subsection{Medical imaging, diagnosis and therapy}

Noble metal nanoparticles composed of gold or silver can behave as non-toxic carriers for medical applications. Noble metal nanoparticles exhibit strong absorption and scattering in the visible and near infrared regions because of surface plasmon resonance (SPR) [63]. Light absorption occurs when the photon energy is dissipated by electron oscillation in the nanoparticle, which is efficiently converted into heat. Noble metal nanoparticles have been used as selective photothermal agents to target cancer cells upon heating; the activity of the nanoparticles to behave as such agents was affected by the size of the nanoparticles $[3,64]$. Light scattering occurs when the photon causes electron oscillation in the nanoparticle, which then emits photons in the form of scattered light either at the same frequency as the incident light (Rayleigh scattering) or at a different frequency (Raman scattering). The structure and size of metal nanoparticles affects the images obtained [65]. The basic concepts of the activity of noble metal nanoparticles for use in imaging, diagnosis and thermal therapy applications are illustrated in Figure 3.

SPR scattering images and SPR absorption spectra have been obtained for gold nanoparticles conjugated to monoclonal anti-epidermal growth factor receptor (anti-EGFR) antibodies after respective incubation in cell cultures containing a nonmalignant epithelial cell line $(\mathrm{HaCaT})$ and two

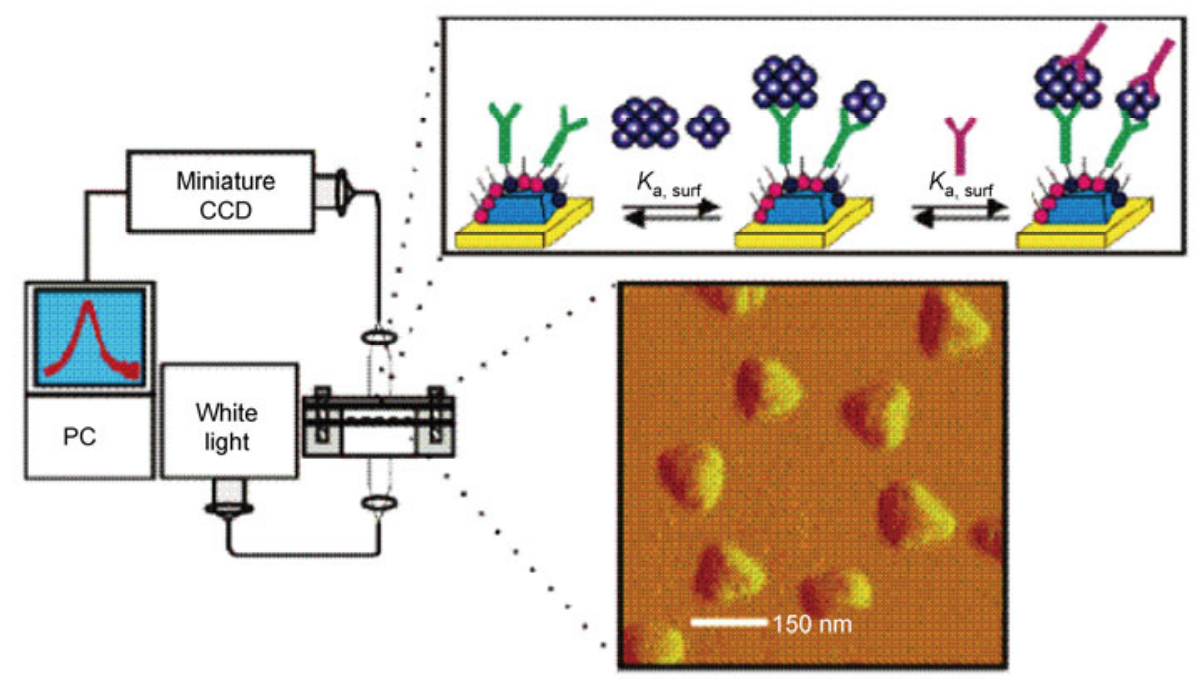

Figure 2 (Color online) Design and experimental setup of an LSPR biosensor for detecting ADDLs using a sandwich assay. Transmission UV-vis spectroscopy was used to monitor the optical properties (LSPR) of Ag nanoparticles. The schematic illustration displays the sandwich assay and surface chemistry of the LSPR nanosensor. First, surface-confined Ag nanoparticles are synthesized on mica substrates using NSL. Next, a self-assembled monolayer consisting of a mixture of 1-OT and MUA is passivated to the nanoparticles through nonspecific binding, which activates the nanoparticles to allow the first anti-ADDL antibody to attach. Finally, an enhancement of the LSPR shift is detected in response to ADDL binding [5]. 


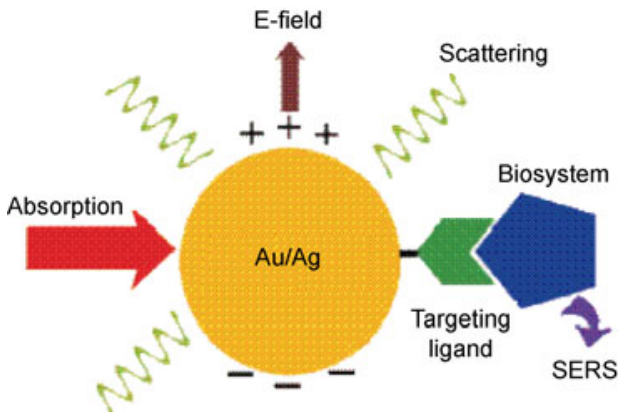

Figure 3 (Color online) The basic concepts of the activity of noble metal nanoparticles for use in medical applications [66].

malignant oral epithelial cell lines (HOC 313 clone 8 and HSC 3) [4]. Specific and homogeneous binding was found to give a sharper, red-shifted SPR absorption band compared with that observed when the system was added to noncancerous cells (see Figure 4). This suggests that SPR scattering images or SPR absorption spectra generated from antibody-conjugated gold nanoparticles can be useful for medical imaging, diagnosis and investigation.

The capability of plasmonic gold nanoparticles and their conjugates to convert absorbed light to heat suggests that they can be used as selective photothermal agents in molecular cancer cell targeting. It has been shown that malignant cells are killed by less than half the laser energy required to kill benign cells after incubation with the anti-EGFR antibody-Au nanoparticle system (see Figure 5) [3].

\section{Conclusion}

This review has summarized recent studies on the development of noble metal nanoparticle-biomolecule conjugates for optical applications. Functionalization of nanoparticles with biomolecules is based on four main approaches, including electrostatic adsorption, direct chemisorption of
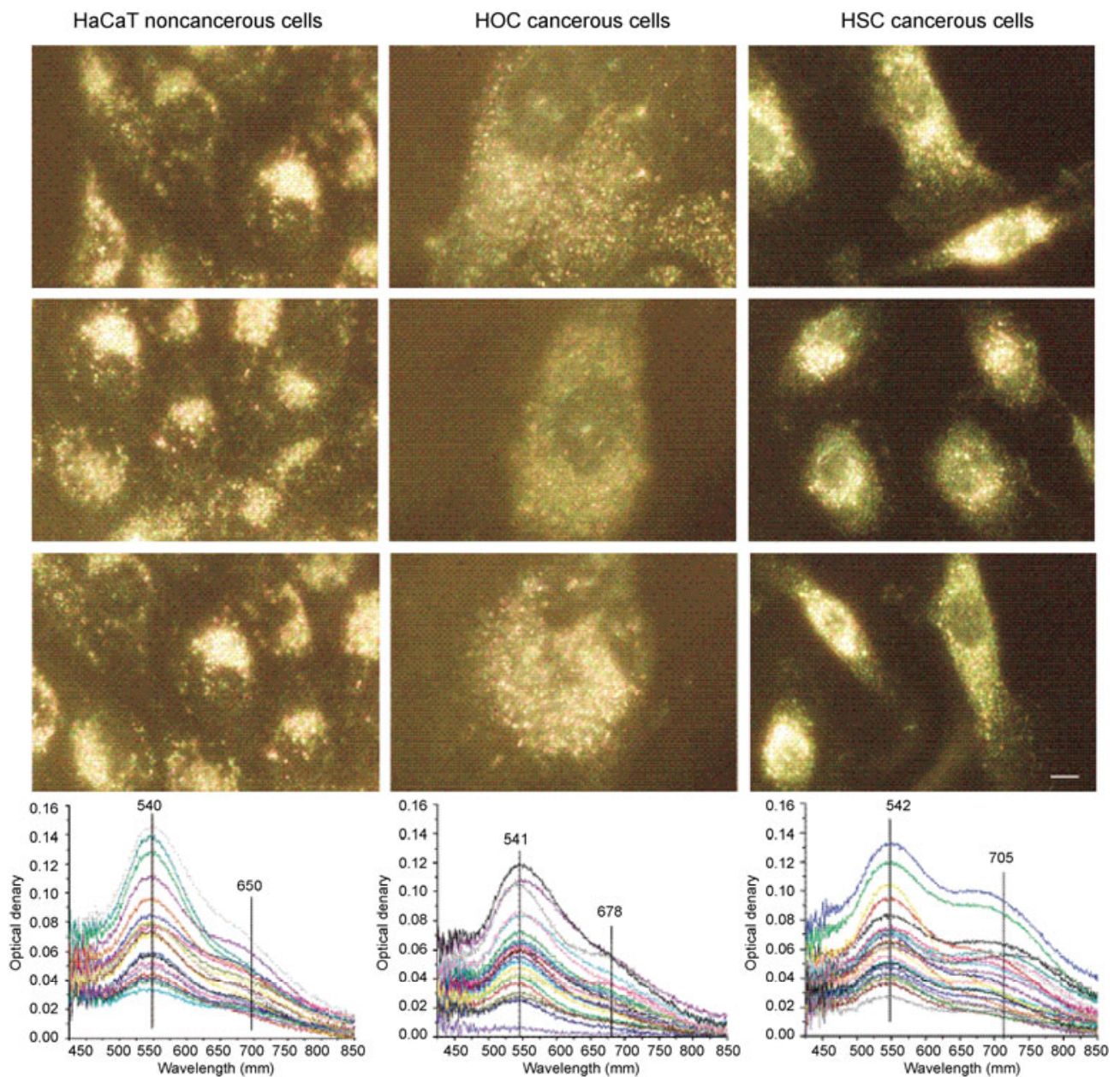

Figure 4 (Color online) Two simple techniques for sensitive cancer diagnosis using anti-EGFR/Au-nanoparticle conjugates: light scattering images (top three rows) and microabsorption spectra (bottom row) are shown for HaCaT noncancerous cells (left column), HOC cancerous cells (middle column), and HSC cancerous cells (right column) after incubation with the nanoparticle conjugate. Three different images of each kind of cells are shown for reproducibility. Microabsorption spectra were measured for 25 different cells. Differences can be seen in the scattering images and absorption spectra obtained for noncancerous (left column) and cancerous cells (right two columns). Specific binding of the nanoparticle systems to the surface of the cancer cells occurs in higher concentration than to the noncancerous cells. Scale bar $=10 \mu \mathrm{m}[4]$. 

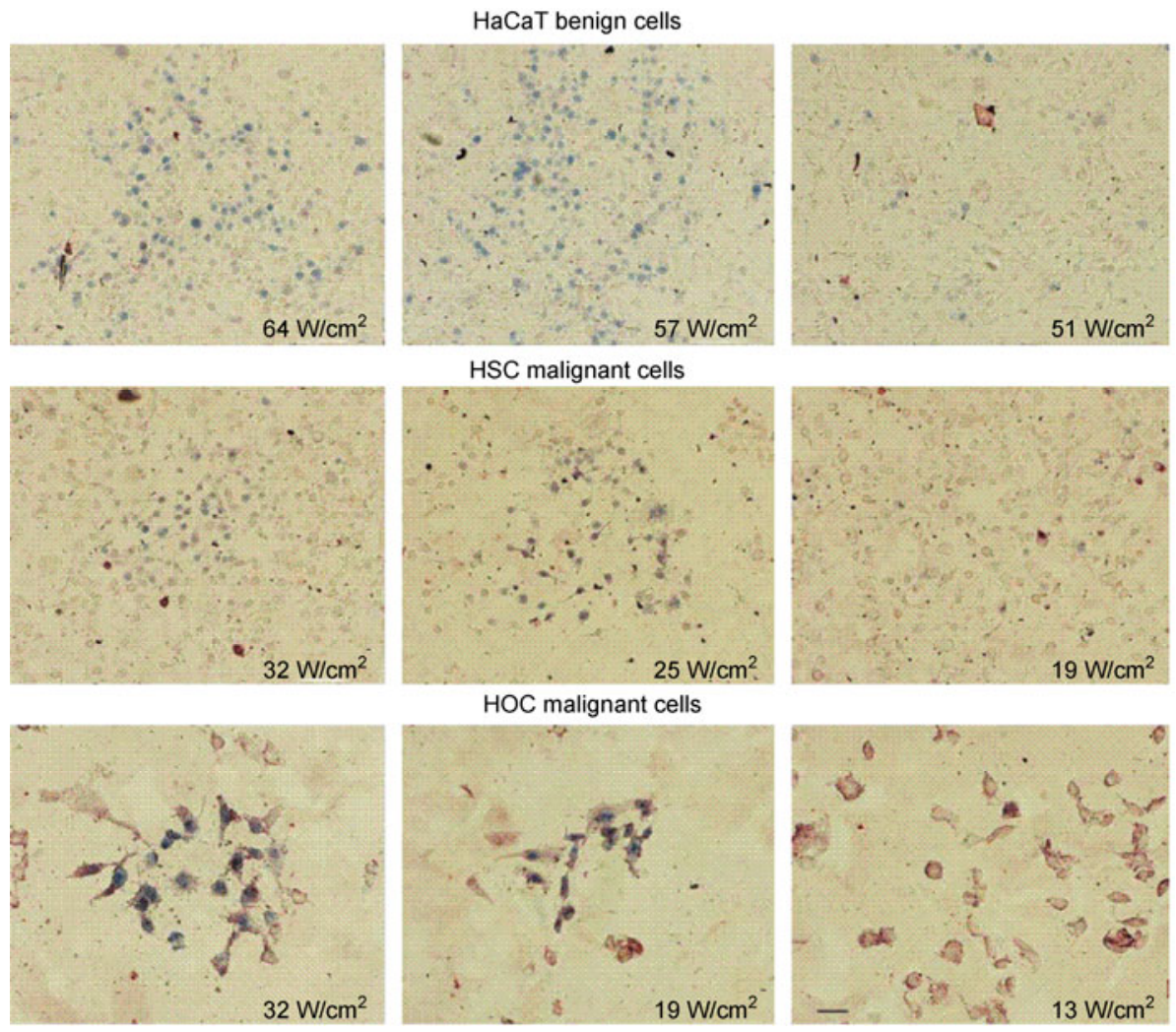

Figure 5 (Color online) Microscope images of benign HaCaT cells (top row), malignant HSC cells (middle row), and malignant HOC cells (bottom row) irradiated at different laser powers and then stained with trypan blue. These images show selective photothermal damage of cancer cells when the anti-EGFR/Au-nanopar- ticle conjugate is used. Benign HaCaT cells are killed at and above $57 \mathrm{~W} / \mathrm{cm}^{2}$, malignant $\mathrm{HSC}$ cells are killed at and above $25 \mathrm{~W} / \mathrm{cm}^{2}$, and malignant HOC cells are killed at and above $19 \mathrm{~W} / \mathrm{cm}^{2}$. Scale bar $=60 \mu \mathrm{m} \mathrm{[3]}$.

thiol derivatives, covalent binding through bifunctional linkers and specific affinity interactions. The properties and optical applications of such systems, such as optical biosensors, and medical imaging, diagnosis and therapy, have been discussed. Labeling biomolecules, such as proteins, antibodies and DNA, with noble metal nanoparticles is a relatively new field and is developing rapidly. Because most biomolecules that have been labeled with nanoparticles have only received limited research, and the technology of nanoparticle-biomolecule conjugates is not yet sophisticated enough, the labeling of biomolecules with complex functions, and new applications of these systems, will be the focus of the future.

This work was supported by the China-MOST International Science \& Technology Cooperation and Exchange Project (2008DFA51230), National Basic Research Program of China (2007CB936603), and National Natural Science Foundation of China (11074207 and 60776007).

1 Pengo P, Baltzer L, Pasquato L, et al. Substrate modulation of the activity of an artificial nanoesterase made of peptide-functionalized gold nanoparticles. Angew Chem Int Ed, 2007, 46: 400-406

2 Xu Z P, Zeng Q H, Lu G Q, et al. Inorganic nanoparticles as carriers for efficient cellular delivery. Chem Eng Sci, 2006, 61: 1027- 1040

3 El-Sayed I H, Huang I X, El-Sayed M A. Selective laser photothermal therapy of epithelial carcinoma using anti-EGFR antibody conjugated gold nanoparticles. Cancer Lett, 2006, 239: 129-135

4 El-Sayed I H, Huang X H, El-Sayed M A. Surface plasmon resonance scattering and absorption of anti-EGFR antibody conjugated gold nanoparticles in cancer diagnostics: Applications in oral cancer. Nano Lett, 2005, 5: 829-834

5 Haes A J, Chang L, Klein W L, et al. Detection of a biomarker for alzheimer's disease from synthetic and clinical samples using a nanoscale optical biosensor. J Am Chem Soc, 2005, 127: 2264-2271

6 Nykypanchuk D, Maye M M, van der Lelie D, et al. DNA-guided crystallization of colloidal nanoparticles. Nature, 2008, 451: 549-552

7 Crespilho F N, Lima F C A, da Silva A B F, et al. The origin of the molecular interaction between amino acids and gold nanoparticles: A theoretical and experimental investigation. Chem Phys Lett, 2009, 469: 186-190

8 EI-Sayed M A. Small is different: Shape-, size-, and compositiondependent properties of some colloidal semiconductor nanocrystals. Acc Chem Res, 2004, 37: 326-333

9 Endo T, Kerman K, Nagatani N, et al. Excitation of localized surface plasmon resonance using a core-shell structured nanoparticle layer substrate and its application for label-free detection of biomolecular interactions. J Phys: Condens Matter, 2007, 19: 215201

10 Love C S, Ashworth I, Brennan C, et al. Dendron-protected au nanoparticles-effect of dendritic structure on chemical stability. J Coll Interface Sci, 2006, 302: 178-186

11 Mauriz E, Calle A, Montoya A, et al. Determination of environmental organic pollutants with a portable optical immunosensor. Talanta, 2006, 69: 359-364

12 Zhang X Y, Young M A, Lyandres O, et al. Rapid detection of an anthrax biomarker by surface-enhanced raman spectroscopy. J Am Chem Soc, 2005, 127: 4484-4489

13 Maxwell D J, Taylor J R, Nie S. Self-assembled nanoparticle probes 
for recognition and detection of biomolecules. J Am Chem Soc, 2002, 124: 9606-9612

14 Rosi N L, Giljohann D A, Thaxton C S, et al. Oligonucleotide-modified gold nanoparticles for intracellular gene regulation. Science, 2006, 312: 1027-1030

15 Chen S H, Carroll D L. Synthesis and characterization of truncated triangular silver nanoplates. Nano Lett, 2002, 2: 1003-1007

16 Metraux G S, Mirkin C A. Rapid thermal synthesis of silver nanoprisms with chemically tailorable thickness. Adv Mater, 2005, 17: $412-415$

17 Sau T K, Murphy C J. Room temperature, high-yield synthesis of multiple shapes of gold nanoparticles in aqueous solution. J Am Chem Soc, 2004, 126: 8648-8649

18 Jin R C, Cao Y C, Hao E C, et al. Controlling anisotropic nanoparticle growth through plasmon excitation. Nature, 2003, 425: 487-490

19 Yin B S, Ma H Y, Wang S Y, et al. Electrochemical synthesis of silver nanoparticles under protection of poly( $\mathrm{N}$-vinylpyrrolidone). J Phys Chem B, 2003, 107: 8898-8904

20 Foss C A Jr, Hornyak G L, Stockert J A, et al. Template-synthesized nanoscopic gold particles: Optical spectra and the effects of particle size and shape. J Phys Chem, 1994, 98: 2963-2971

21 Jin R, Egusa S, Scherer N F. Thermally-induced formation of atomic Au clusters and conversion into nanocubes. J Am Chem Soc, 2004, 126: 9900-9901

22 Sun Y G, Xia Y N. Shape-controlled synthesis of gold and silver nanoparticles. Science, 2002, 298: 2176-2179

23 Zhu S L, Li F, Du C L, et al. A localized surface plasmon resonance nanosensor based on rhombic Ag nanoparticle array. Sensors Actuators B, 2008 134: 193-198

24 Haes A J, Van Duyne R P. A nanoscale optical biosensor: Sensitivity and selectivity of an approach based on the localized surface plasmon resonance spectroscopy of triangular silver nanoparticles. J Am Chem Soc, 2002, 124: 10596-10604

25 Shen X C, Yuan Q, Liang H, et al. Hysteresis effects of the interaction between serum albumins and silver nanoparticles. Sci China: Ser B, 2003, 46: 387-398

26 Haes A J, Hall W P, Chang L, et al. A localized surface plasmon resonance biosensor: first steps toward an assay for alzheimer's disease. Nano Lett, 2004, 4: 1029-1034

27 Katz E, Willner I. Integrated nanoparticle-biomolecule hybrid systems: synthesis, properties, and applications. Angew Chem Int Ed, 2004, 43: 6042-6108

28 Aubin-Tam M-E, Hamad-Schifferli K. Structure and function of nanoparticle-protein conjugates. Biomed Mater, 2008, 3: 034001-034017

29 Willner I, Baron R, Willner B. Integrated nanoparticle-biomolecule systems for biosensing and bioelectronics. Biosensors Bioelectron, 2007, 22: 1841-1852

30 Pingarron J M, Yanez-Sedeno P, Gonzalez-Cortes A. Gold nanoparticle-based electrochemical biosensors. Electrochimica Acta, 2008, 53: 5848-5866

31 Guo S, Dong S. Biomolecule-nanoparticle hybrids for electrochemical biosensors. Trends Anal Chem, 2009, 28: 96-109

$32 \mathrm{Lu} \mathrm{Y,} \mathrm{Liu} \mathrm{J.} \mathrm{Catalyst-functionalized} \mathrm{nanomaterials.} \mathrm{Wires} \mathrm{Nanomed}$ Nanobi, 2009, 1: 35-46

33 Li D, Song S P, Fan C H. Target-responsive structural switching for nucleic acid-based sensors. Acc Chem Res, 2010, 43: 631-641

34 Song S P, Qin Y, He Y, et al. Functional nanoprobes for ultrasensitve detection of biomolecules. Chem Soc Rev, 2010, 39: 4234-4243

35 Roca M, Haes A J. Probing cells with noble metal nanoparticle aggregates. Nanomedicine, 2008, 3: 555-565

36 Ghosh P, Han G, De M, et al. Gold nanoparticles in delivery applications. Adv Drug Delivery Rev, 2008, 60: 1307-1315

37 Baptista P, Pereira E, Eaton P, et al. Gold nanoparticles for the development of clinical diagnosis methods. Anal Bioanal Chem, 2008, 391: 943-950

38 He Y, Fan C H, Lee S T. Silicon nanostructures for bioapplications. Nano Today, 2010, 5: 282-295

39 Johnston A P R, Cortez C, Angleatos A S, et al. Layer-by-layer engineered capsules and their applications. Curr Opinion Coll \& Inter- face Sci, 2006, 11: 203-209

40 Angelatos A S, Radt B, Caruso F. Light-responsive polyelectrolyte/ gold nanoparticle microcapsules. J Phys Chem B, 2005, 109: 3071-3076

41 Zhao W B, Park J, Caminade A-M, et al. Localized surface plasmon resonance coupling in Au nanoparticles/phosphorus dendrimer multilayer thin films fabricated by layer-by-layer self-assembly method. J Mater Chem, 2009, 19: 2006-2012

42 Yu A M, Liang Z J, Cho J H, et al. Nanostructured electrochemical sensor based on dense gold nanoparticle films. Nano Lett, 2003, 3: 1203-1207

43 Shen X C, Liu X Y, Liang H, et al. Spectroscopic studies of interaction between bovine hemoglobin and Ag nanoparticles. Acta Chim Sin, 2006, 64: 469-474

44 Du P, Li H X, Mei Z H, et al. Electrochemical DNA biosensor for the detection of DNA hybridization with the amplication of Au nanoparticles and CdS nanoparticles. Bioelectrochemistry, 2009, 75: 37-43

45 Xiang C L, Zou Y J, Sun L X, et al. Direct electrochemistry and electrocatalysis of cytochrome immobilized on gold nanoparticleschitosan-carbon nanotubes-modified electrode. Talanta, 2007, 74: 206-211

46 Cederquist K B, Keating C D. Curvature effects in DNA:Au nanoparticle conjugates. ACS Nano, 2009, 3: 256-260

47 Cao Y W, Jin R C, Mirkin C A. Nanoparticles with raman spectroscopic fingerprints for DNA and RNA detection. Science, 2002, 297: $1536-1540$

48 Mirkin C A, Letsinger R L, Mucic R C, et al. A DNA-based method for rationally assembling nanoparticles into macroscopic materials. Nature, 1996, 382: 607-609

49 Elghanian R, Storhoff J J, Mucic R C, et al. Selective colorimetric detection of polynucleotides based on the distance-dependent optical properties of gold nanoparticles. Science, 1997, 277: 1078-1081

50 Riboh J C, Haes A J, McFarland A D, et al. A nanoscale optical biosensor: real-time immunoassay in physiological buffer enabled by improved nanoparticle adhesion. J Phys Chem B, 2003, 107: $1772-1780$

51 Nath N, Chilkoti A. A colorimetric gold nanoparticle sensor to interrogate biomolecular interactions in real time on a surface. Anal Chem, 2002, 74: 504-509

52 Huang T, Nallathamby P D, Gillet D, et al. Design and synthesis of single nanoparticle optical biosensors for imaging and characterization of single receptor molecules on single living cells. Anal Chem, 2007, 79: 7708-7718

53 Shenton W, Davis S A, Mann S. Directed self-assembly of nanoparticles into macroscopic materials using antibody-antigen recognition. Adv Mater, 1999, 11: 449-452

54 Eustis S, El-Sayed M A. Why gold nanoparticles are more precious than pretty gold: Noble metal surface plasmon resonance and its enhancement of the radiative and nonradiative properties of nanocrystals of different shapes. Chem Soc Rev, 2006, 35: 209-217

55 Malinsky M D, Kelly K L, Schatz G C, et al. Chain length dependence and sensing capabilities of the localized surface plasmon resonance of silver nanoparticles chemically modified with alkanethiol self-assembled monolayers. J Am Chem Soc, 2001, 123: 1471-1482

56 Haynes C L, Yonzon C R, Zhang X, et al. Surface-enhanced raman sensors: Early history and the development of sensors for quantitative biowarfare agent and glucose detection. J Raman Spectrosc, 2005, 36: 471-484

57 Michaels A M, Nirmal M, Brus L E. Surface enhanced raman spectroscopy of individual rhodamine $6 \mathrm{G}$ molecules on large $\mathrm{Ag}$ nanocrystals. J Am Chem Soc, 1999, 121: 9932-9939

58 Schatz G C, Van Duyne R P. Electromagnetic Mechanism of Surface-enhanced Spectroscopy. New York: John Wiley \& Sons Ltd., 2002

59 Xie F, Baker M S, Goldys E M. Homogeneous silver-coated nanoparticle substrates for enhanced fluorescence detection. J Phys Chem B, 2006, 110: 23085-23091

60 Li X, Jiang L, Zhan Q Q, et al. Localized surface plasmon resonance (LSPR) of polyelectrolyte-functionalized gold-nanoparticles for bio- 
sensing. Coll Surf A: Physicochem, 2009, 332: 172-179

61 Mao X, Ma Y Q, Zhang A G, et al. Disposable nucleic acid biosensors based on gold nanoparticle probes and lateral flow strip. Anal Chem, 2009, 81: 1660-1668

62 Shin D O, Jeong J R, Han T H, et al. A plasmonic biosensor array by block copolymer lithography. J Mater Chem, 2010, 20: 7241-7247

63 Willets K A, Van Duyne R P. Localized surface plasmon resonance spectroscopy and sensing. Annu Rev Phys Chem, 2007, 58: 269-297

64 Moran C H, Wainerdi S M, Cherukuri T K, et al. Size-dependent joule heating of gold nanoparticles using capacitively coupled radiofrequency fields. Nano Res, 2009, 2: 400-405

65 Tao A, Sinsermsuksakul P, Yang P D. Polyhedral silver nanocrystals with distinct scattering signatures. Angew Chem Int Ed, 2006, 45: 4597-4601

66 Jain K P, Huang X H, El-Sayed H I, et al. Noble metals on the nanoscale: optical and photothermal properties and some applications in imaging, sensing, biology, and medicine. Acc Chem Res, 2008, 41: $1578-1586$

Open Access This article is distributed under the terms of the Creative Commons Attribution License which permits any use, distribution, and reproduction in any medium, provided the original author(s) and source are credited. 